
\title{
Research Suare \\ Ultra-Sensitive Detection of Pyridine in Water Using Zinc Porphyrin Incorporated in a Transparent Hydrophobic Film
}

Ryo Sasai ( $\nabla$ rsasai@riko.shimane-u.ac.jp )

Shimane University

Yu-hei Aoyama

Shimane University

Takuya Fujimura

Shimane University

\section{Research Article}

Keywords:

Posted Date: December 14th, 2021

DOI: https://doi.org/10.21203/rs.3.rs-1068121/v2

License: (c) (i) This work is licensed under a Creative Commons Attribution 4.0 International License.

Read Full License

Version of Record: A version of this preprint was published at Scientific Reports on April 6th, 2022. See the published version at https://doi.org/10.1038/s41598-022-09927-x. 


\title{
Ultra-Sensitive Detection of Pyridine in Water Using Zinc Porphyrin Incorporated in a Transparent Hydrophobic Film
}

Authors:

\author{
Ryo Sasai*, Yu-hei Aoyama, \& Takuya Fujimura*
}

\begin{abstract}
Affiliation:
Graduate School of Natural Science and Technology, Shimane University, 1060 Nishi-Kawatsu-cho, Matsue 690-8504, Japan
\end{abstract}

Correspondence should be addressed to R. S. (email: rsasai@riko.shimane-u.ac.jp) or to T. F. (email: tfujimura@riko.shiamene-u.ac.jp)

\begin{abstract}
In this study, we investigated the axial coordination reaction between pyridine and zinc mesotetra(4-sulfonatophenyl)porphyrin (ZnTPPS) incorporated in a transparent layered double hydroxide (LDH) film modified with 1-decanesulfonate (C10S) in an aqueous solution. The equilibrium constant $\left(K_{11}\right)$ of the axial coordination reaction between pyridine and ZnTPPS incorporated in the transparent ZnTPPS/C10S/LDH film was approximately 260 times that of the corresponding reaction in an aqueous solution. The hydrophobisation of the LDH interlayer space by $\mathrm{C} 10 \mathrm{~S}$, which led to the elimination of water molecules surrounding ZnTPPS and enabled the accumulation of pyridine molecules, was responsible for such a significant increase in the $K_{11}$ value. The developed film can detect pyridine in aqueous solutions with ultra-high sensitivity in the order of $10^{-5} \mathrm{~mol} / \mathrm{L}$ through changes in the colour tone, which is comparable to the molecular detection ability of insect antennae. The sensing response was also observed at pyridine concentrations as low as $10^{-9} \mathrm{~mol} / \mathrm{L}$.
\end{abstract}

\section{Introduction}

Sensor devices that can detect specific chemical compounds with high sensitivity and selectivity are key to achieving the Sustainable Development Goals adopted by the United Nations in 2015. ${ }^{1}$ Advanced sensors that can detect extremely small amounts of specific chemicals (at nano- and micromolar concentrations) that are difficult to identify using current sensors, are desirable in various fields, such as non-invasive biological and environmental monitoring. Although sensors based on quartz oscillators and semiconductors that show ultra-high sensitivities towards various chemicals have been developed, the sensors themselves exhibit limited sensitivities and selectivities, and the range of 
detectable chemicals is not sufficiently diverse. $^{2}$ In this study, we wished to focus on metalloporphyrins, which have advantageous sensing properties. More specifically, metalloporphyrins exhibit spectral changes depending on the attachment of axial ligands to the central metal and the coordinated ligand species, ${ }^{2}$ wherein the ease of coordination depends on the central metal species. ${ }^{2}$ Thus, the spectroscopic changes caused by coordination can be utilized to achieve the highly sensitive detection of chemicals in various media. ${ }^{2}$ However, the detection of target chemicals in aqueous media is impeded by the competition between the axial coordination reactions of the target chemicals with the central metal of the metalloporphyrin in water, in addition to the reactions between the target chemicals and water. In such systems, the detection limit can be improved via the hydrophobisation of the metalloporphyrin environment.

In this context, we have previously developed functional materials by hybridizing functional molecules, such as dyes, with ion-exchangeable layered inorganic compounds modified with amphipathic molecules. ${ }^{4-20}$ More specifically, an R6G/C16TMA/Lap hybrid material comprising rhodamine 6G (R6G) and laponite clay (Lap) modified by the hexadecyltrimethylammonium (C16TMA) cation, retained the luminous properties of R6G under both dry and wet conditions. ${ }^{6}$ Through the use of hexyltrimethylammonium (C6TMA) cations with shorter alkyl chains instead of C16TMA, the luminous properties of R6G in the R6G/C6TMA/Lap hybrid material varied under dry and wet conditions. ${ }^{7}$ These results indicate that the adsorption of water molecules in the interlayer space of Lap modified by alkyltrimethylammonium cations can be controlled by tuning the hydrophobicity of amphipathic molecules. Therefore, the axial coordination reaction of the target chemicals with the central metal of the metalloporphyrin can be expected to proceed in the interlayer space of ion-exchangeable layered inorganic compounds modified with amphipathic molecules bearing long alkyl chains without competition from water molecules.

Herein, we report the preparation of a transparent solid film by hybridizing zinc-meso-tetra(4sulfonatophenyl)porphyrin (ZnTPPS) and 1-decanesulfonate (C10S) with a layered double hydroxide (LDH), which is a layered inorganic compound similar to anion-exchangeable clay. Hereafter, we refer to the prepared transparent solid film as the ZnTPPS/C10S/LDH transparent solid film. Subsequently, we immersed the film in aqueous pyridine solutions of various concentrations and investigated the axial coordination reaction between pyridine and ZnTPPS in the ZnTPPS/C10S/LDH transparent solid film by UV-Vis absorption spectroscopy.

\section{Results and Discussion}



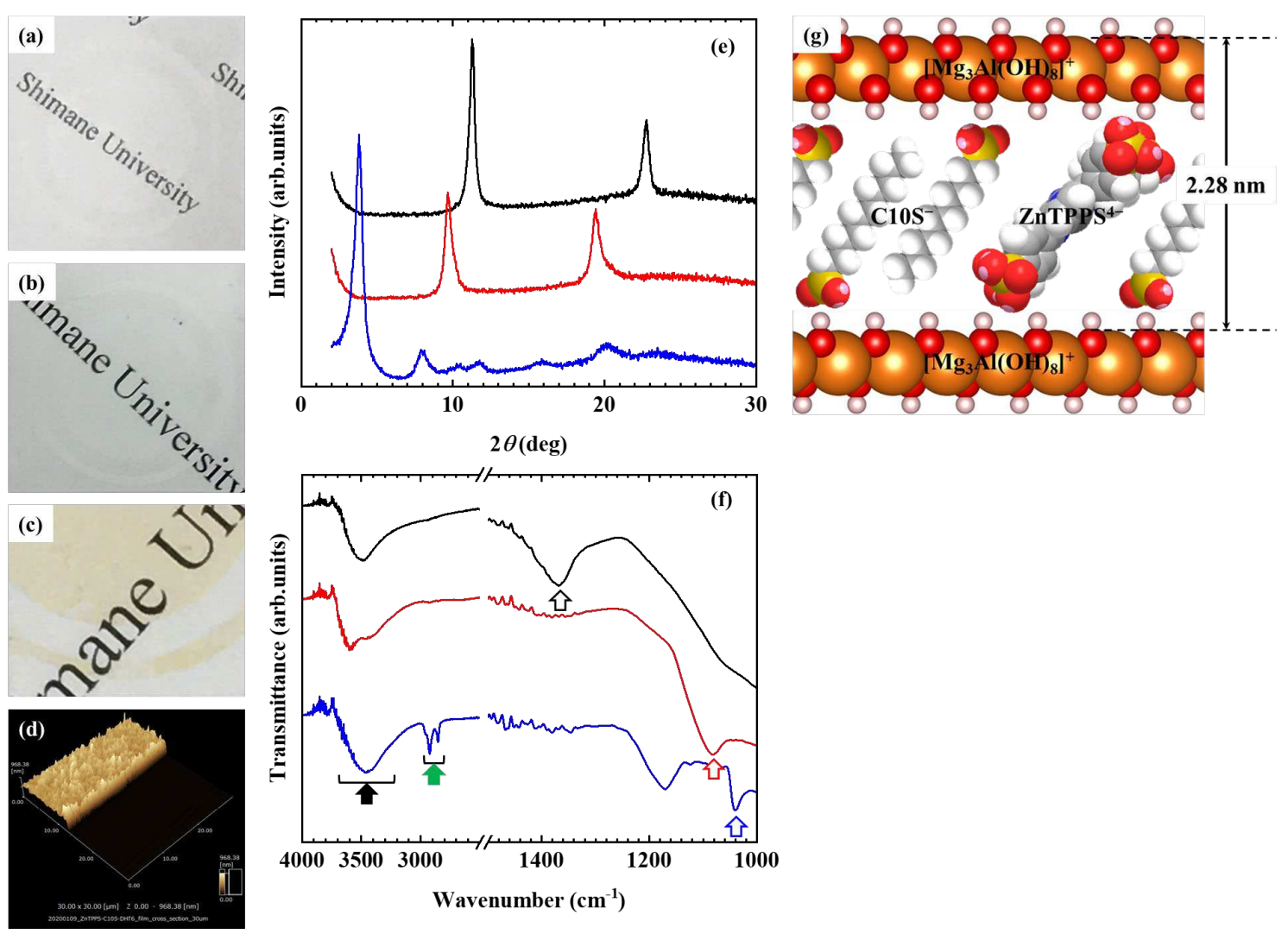

Figure 1. Photographic images of the (a) $\mathrm{CO}_{3}{ }^{2-}-\mathrm{LDH}$, (b) $\mathrm{ClO}_{4}{ }^{-}-\mathrm{LDH}$, and (c) ZnTPPS/C10S/LDH transparent films. (d) AFM image of the transparent ZnTPPS/C10S/LDH film. (e) XRD patterns of the $\mathrm{CO}_{3}{ }^{2-}$-LDH (black solid line), $\mathrm{ClO}_{4}{ }^{-}-\mathrm{LDH}$ (red solid line), and ZnTPPS/C10S/LDH transparent films (blue solid line). (h) FT-IR spectra of the $\mathrm{CO}_{3}{ }^{2-}-\mathrm{LDH}$ (black solid line), $\mathrm{ClO}_{4}{ }^{-}$-LDH (red solid line), and transparent ZnTPPS/C10S/LDH films (blue solid line). Arrows indicate the peaks originating from the hydroxyl (black), carbonate (black (open)), methylene (green), perchlorate (red (open)) and sulfonate (blue (open)) groups. (g) Schematic structural model of the ZnTPPS/C10S/LDH materials.

As shown in Figure 1, all the films prepared using the procedures described in this study exhibited sufficient transparency. The incorporation of ZnTPPS in the transparent ZnTPPS/C10S/LDH film was confirmed by the appearance of a light orange colour. Furthermore, the fraction of ZnTPPS incorporated in the transparent ZnTPPS/C10S/LDH film was estimated to be $3.8 \%$ based on an analysis of the anion-exchange capacity of the immersion solution obtained after the immersing operation. According to the results of atomic force microscopy (AFM) studies, the average thickness of the transparent ZnTPPS/C10S/LDH film was $579.3 \mathrm{~nm}$. From the X-ray diffraction (XRD) patterns of the $\mathrm{CO}_{3}{ }^{2-}-\mathrm{LDH}, \mathrm{ClO}_{4}{ }^{-}-\mathrm{LDH}$, and transparent ZnTPPS/C10S/LDH films (Figure 1(e)), the $d_{003}$ values of the $\mathrm{CO}_{3}{ }^{2-}-\mathrm{LDH}, \mathrm{ClO}_{4}{ }^{-}-\mathrm{LDH}$, and transparent $\mathrm{ZnTPPS} / \mathrm{C} 10 \mathrm{~S} / \mathrm{LDH}$ films were found to be $0.785,0.954$, and $2.28 \mathrm{~nm}$, respectively, indicating that the target anions were incorporated in the 
interlayer spaces of the LDHs in the transparent films. In addition, the Fourier-transform infrared (FTIR) spectra of the $\mathrm{CO}_{3}{ }^{2-}-\mathrm{LDH}, \mathrm{ClO}_{4}^{-}-\mathrm{LDH}$, and $\mathrm{ZnTPPS} / \mathrm{C} 10 \mathrm{~S} / \mathrm{LDH}$ transparent films are shown in Figure 1(f). The absorption band at $1368 \mathrm{~cm}^{-1}$ corresponding to the carbonate anions of the transparent $\mathrm{CO}_{3}{ }^{2-}$ - $\mathrm{LDH}$ film was absent in that of the spectrum for the transparent $\mathrm{ClO}_{4}{ }^{-}-\mathrm{LDH}$ film; instead, an absorption band ascribed to the perchlorate anions was observed. Moreover, in the FT-IR spectrum of the transparent ZnTPPS/C10S/LDH film, absorption bands corresponding to the hydroxyl groups from the LDH layer surface and the hydrated water molecules appeared at $\sim 3500 \mathrm{~cm}^{-1}$, while bands originating from the methylene groups of C10S appeared at 2923 and $2855 \mathrm{~cm}^{-1}$, and those attributed to the sulfonate groups of C10S and ZnTPPS were observed at $1040 \mathrm{~cm}^{-1}$. These results indicated the successful synthesis of the transparent ZnTPPS/C10S/LDH film by the procedure reported herein. The schematic structural model of ZnTPPS/C10S/LDH shown in Figure 1(g) was therefore proposed based on the molecular sizes of ZnTPPS $(1.90 \mathrm{~nm})$ and C10S $(1.58 \mathrm{~nm})$, the thickness of the LDH monolayer $(0.48 \mathrm{~nm})$, and the $d_{003}$ value $(2.28 \mathrm{~nm})$.
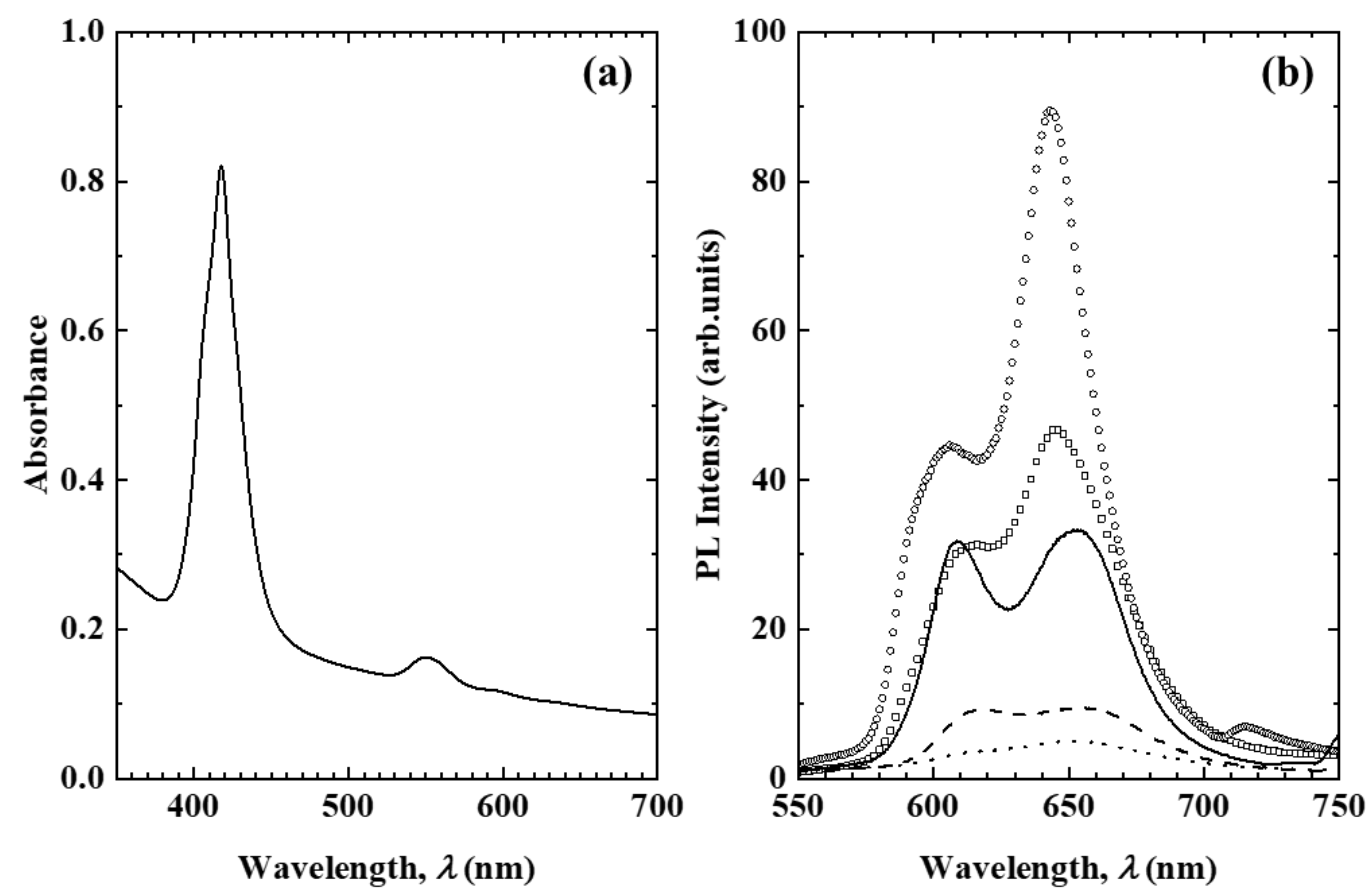

Figure 2. (a) Absorption spectrum of the transparent ZnTPPS/C10S/LDH film. (b) PL spectra of the transparent ZnTPPS/C10S/LDH film at excitation wavelengths of $410(\square), 420(\bigcirc), 430$ (solid line), 440 (broken line), and 450 (dotted line) $\mathrm{nm}$.

Figure 2 shows the absorption and photoluminescence (PL) spectra of the transparent ZnTPPS/C10S/LDH film. The Soret and Q bands originating from ZnTPPS in the absorption spectrum of the transparent ZnTPPS/C10S/LDH film (Figure 2(a)) indicated that the majority of ZnTPPS molecules incorporated in the interlayer space of the LDH in the transparent film existed as monomers. 
Furthermore, the PL spectra of the transparent ZnTPPS/C10S/LDH films showed different shapes depending on the excitation wavelength, thereby indicating that a fraction of the ZnTPPS incorporated in the interlayer space of the LDH in the transparent film formed aggregates.

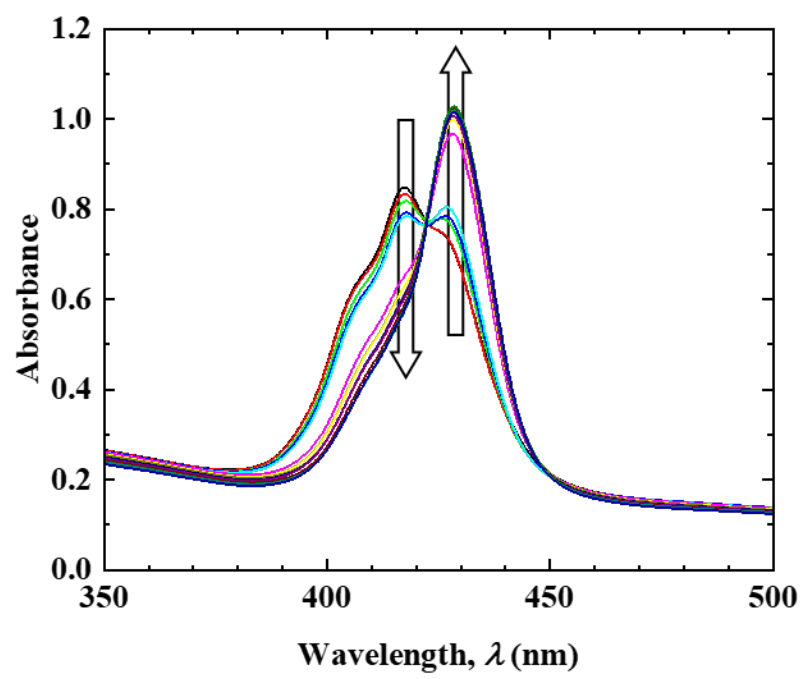

Figure 3. Absorption spectra of the ZnTPPS/C10S/LDH transparent film after immersion in solutions of various [Pyridine] $([$ Pyridine $]=12.3 \mu \mathrm{mol} / \mathrm{L}$ to $0.123 \mathrm{~mol} / \mathrm{L})$.

Figure 3 depicts the absorption spectra of the transparent ZnTPPS/C10S/LDH film immersed in aqueous pyridine solutions of various concentrations ([Pyridine] ranging from $12.3 \mu \mathrm{mol} / \mathrm{L}$ to 0.123 $\mathrm{mol} / \mathrm{L}$ ). As shown, upon increasing [Pyridine], the intensity of the absorption peak at $417 \mathrm{~nm}$ gradually diminished with the concomitant appearance of a new peak at $428 \mathrm{~nm}$. Moreover, an isosbestic point was observed at $422 \mathrm{~nm}$. Such spectral changes are characteristic of reactions between two states. A similar change in the Soret band was observed during the formation of a 1:1 complex (Py-ZnTPPS) between ZnTPPS and pyridine (Py) as outlined in Equation (1). The reaction was performed in an aqueous medium (Figure S1 in Supporting Information), and upon immersing the transparent ZnTPPS/C10S/LDH film in an aqueous solution of pyridine, the ZnTPPS in the interlayer space of C10S/LDH formed Py-ZnTPPS.

$$
\text { ZnTPPS }+ \text { Py } \rightleftharpoons \text { Py-ZnTPPS }
$$




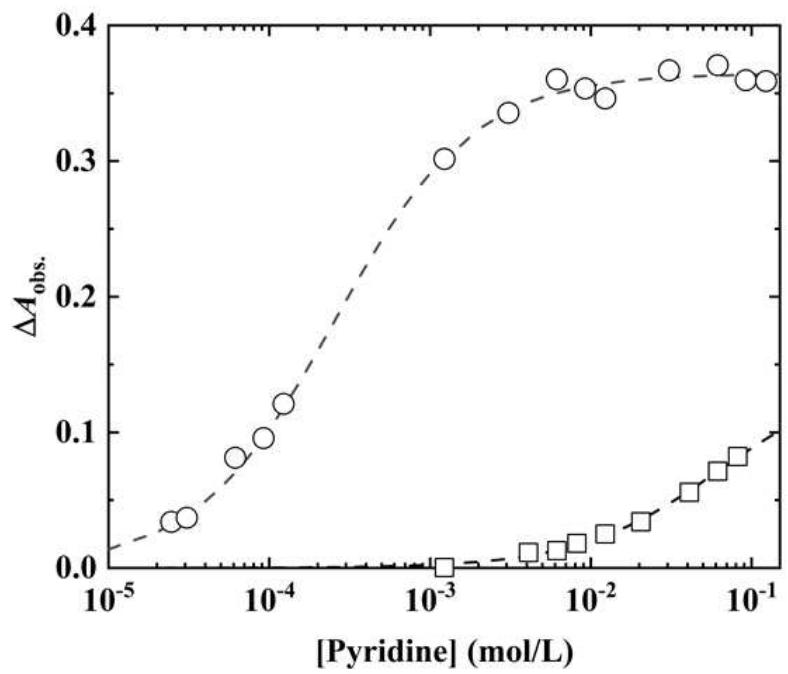

Figure 4. Dependence of $\Delta A_{\text {obs. }}$ on [Pyridine] of the transparent ZnTPPS/C10S/LDH film $(\bigcirc, 429 \mathrm{~nm})$ and ZnTPPS ( $\square, 425 \mathrm{~nm}$ ) in an aqueous solution. The broken lines represent the fitting curves estimated using Equation (1).

The dependence of $\Delta A_{\text {obs. }}\left(\Delta A_{\text {obs. }}=A_{\text {obs. }}-A_{0}\right)$ on [Pyridine] is outlined in Figure 4, where $A_{\text {obs. }}$ and $A_{0}$ are the absorbances corresponding to the Soret bands in the presence and absence of pyridine, respectively. The $\Delta A_{\text {obs. }}$ value of the transparent ZnTPPS/C10S/LDH film was monitored at [Pyridine] values ranging from $10^{-3}$ to $10^{-5} \mathrm{~mol} / \mathrm{L}$ in the aqueous $\mathrm{ZnTPPS}$ solution. The $\Delta A_{\mathrm{obs}}$ values indicate that the rate of formation of the Py-ZnTPPS complex was enhanced by incorporating ZnTPPS in the interlayer space of the LDH modified by C10S. Furthermore, the transparent $\mathrm{ZnTPPS} / \mathrm{C10S} / \mathrm{LDH}$ film was capable of detecting pyridine at low concentrations in the order of $\mu \mathrm{mol}$ per litre. In addition, it was found that the pyridine detection sensitivity of the transparent ZnTPPS/C10S/LDH film in the aqueous solution did not reach that of the Re-based molecular trap (i.e., $72 \mathrm{nmol} / \mathrm{L}$ ) employing an evanescent wave infrared chemical sensing method, which is recognized as a high-sensitivity detection method, and was reported by Huang et al. ${ }^{21}$ However, the pyridine detection sensitivity of the transparent ZnTPPS/C10S/LDH film in the aqueous solution was significantly higher than those of the $\mathrm{ZnS}$ nanoparticles reported by $\mathrm{Li}$ et al. $\left(6.76 \times 10^{-5} \mathrm{~mol} / \mathrm{L}\right)^{22}$ and the $\mathrm{CuI}$-coated $\mathrm{Cu}$ foil reported by $\mathrm{Lv}$ et al. $\left(1.0 \times 10^{-3}\right.$ to $\left.5.0 \mathrm{~mol} / \mathrm{L}\right){ }^{23}$ Therefore, the results presented herein indicate for the first time that extremely sensitive molecular detection can be realised by strategically designing the interlayer space of $\mathrm{LDH}$ to facilitate the axial coordination reaction of metallic porphyrins. Therefore, the developed transparent ZnTPPS/C10S/LDH film serves as a highly sensitive film-based device for pyridine detection.

The dependence of $\Delta A_{\text {obs. }}$ on [Pyridine] can therefore be expressed by Equation (2):

$$
\Delta A_{\text {obs. }}=\frac{b \Delta \varepsilon_{11}}{2 K_{11}}\left[1+K_{11}[\mathrm{Py}]_{0}+K_{11}[\mathrm{ZnTPPS}]_{0}-\left\{\left(1+K_{11}[\mathrm{Py}]_{0}+K_{11}[\mathrm{ZnTPPS}]_{0}\right)^{2}-\right.\right.
$$




$$
\left.\left.4 K_{11}^{2}[\mathrm{Py}]_{0}[\mathrm{ZnTPPS}]_{0}\right\}^{1 / 2}\right]
$$

where

$$
\Delta \varepsilon_{11}=\varepsilon_{11}-\varepsilon_{\mathrm{ZnTPPS}}
$$

where $K_{11}, b,[\mathrm{Py}]_{0},[\mathrm{ZnTPPS}]_{0}, \varepsilon_{11}$, and $\varepsilon_{\mathrm{ZnTPPS}}$ are the equilibrium constants of the PyZnTPPS formation reaction (reaction (1)), light path length $(1 \mathrm{~cm})$, initial [Pyridine], initial concentration of ZnTPPS, and molar extinction coefficients $\left(\mathrm{L} \cdot \mathrm{mol}^{-1} \cdot \mathrm{cm}^{-1}\right)$ of Py-ZnTPPS and ZnTPPS, respectively. To analyse the various data related to the transparent ZnTPPS/C10S/LDH film, uniform dissolution of ZnTPPS in the aqueous solution $(2 \mathrm{~mL})$ was assumed. The [Pyridine] dependence of $\Delta A_{\text {obs. }}$ was analysed by the nonlinear least squares method using Equation (2). A significantly high $K_{11}$ value of $4.17 \times 10^{3} \mathrm{~L} / \mathrm{mol}$ was obtained for the Py-ZnTPPS formation reaction in the transparent ZnTPPS/C10S/LDH film, which was 260 times that for the Py-ZnTPPS formation reaction in the aqueous solution. The formation of Py-ZnTPPS in the aqueous solution competes with the reaction between pyridine and water. Notably, the hydrophobic interlayer space of ZnTPPS/C10S/LDH does not permit water adsorption. In contrast, pyridine is adsorbed and concentrated in the interlayer space of ZnTPPS/C10S/LDH (c.f., Figure 5). Therefore, ZnTPPS can easily form Py-ZnTPPS in the transparent ZnTPPS/C10S/LDH film in the absence of competition from water molecules. As a result, the $K_{11}$ value of the Py-ZnTPPS formation reaction increased significantly.

(a) in water

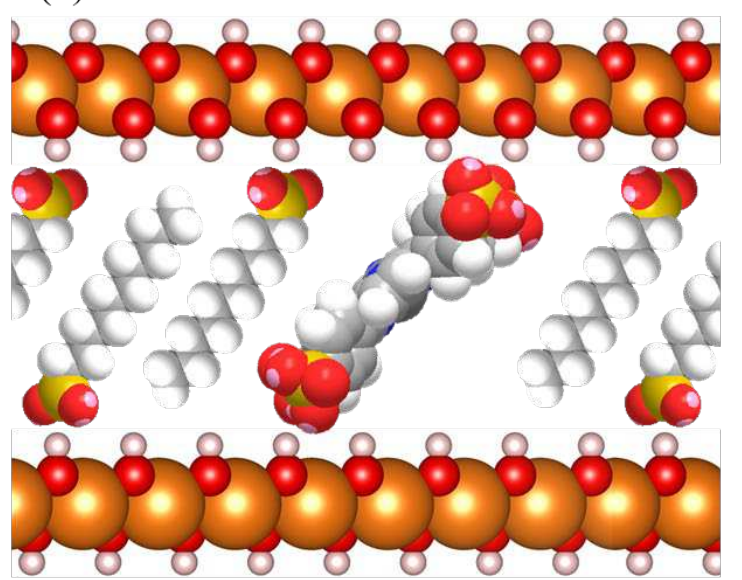

(b) in pyridine aqueous solution

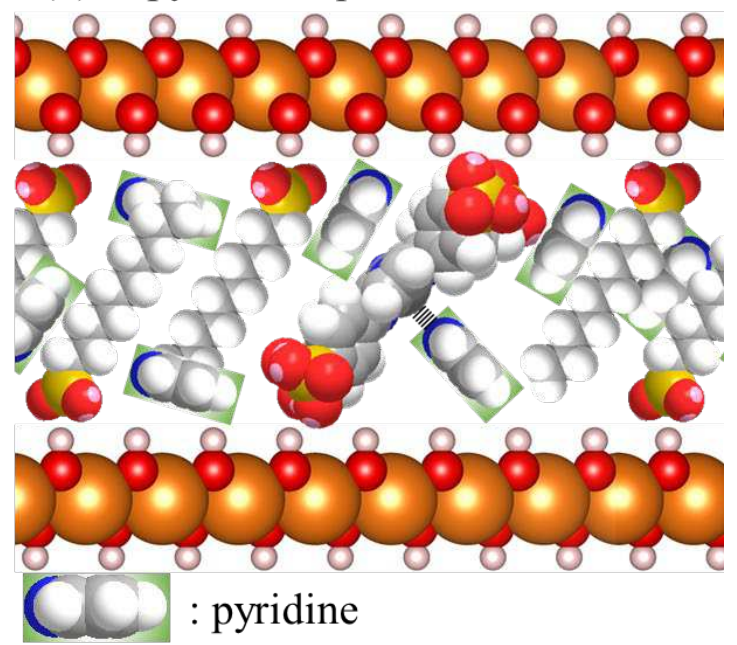

Figure 5. Schematic structural model of the ZnTPPS/C10S/LDH materials in the (a) absence and (b) presence of pyridine in an aqueous solution.

Figure 6(a) shows the absorption spectra of the transparent ZnTPPS/C10S/LDH film immersed in aqueous pyridine solutions with extremely low [Pyridine] (ranging from $1.23 \mathrm{nmol} / \mathrm{L}$ to $12.3 \mu \mathrm{mol} / \mathrm{L}$ ). A change in the absorbance at $417 \mathrm{~nm}$ was noted at a [Pyridine] as low as $1.23 \mathrm{nmol} / \mathrm{L}$. Moreover, the absorbance at $417 \mathrm{~nm}$ decreased with increasing [Pyridine], while the $\Delta A_{\text {obs. }}$ value at $417 \mathrm{~nm}$ decreased exponentially with increasing [Pyridine] (Figure 6(b)). According to these results, 

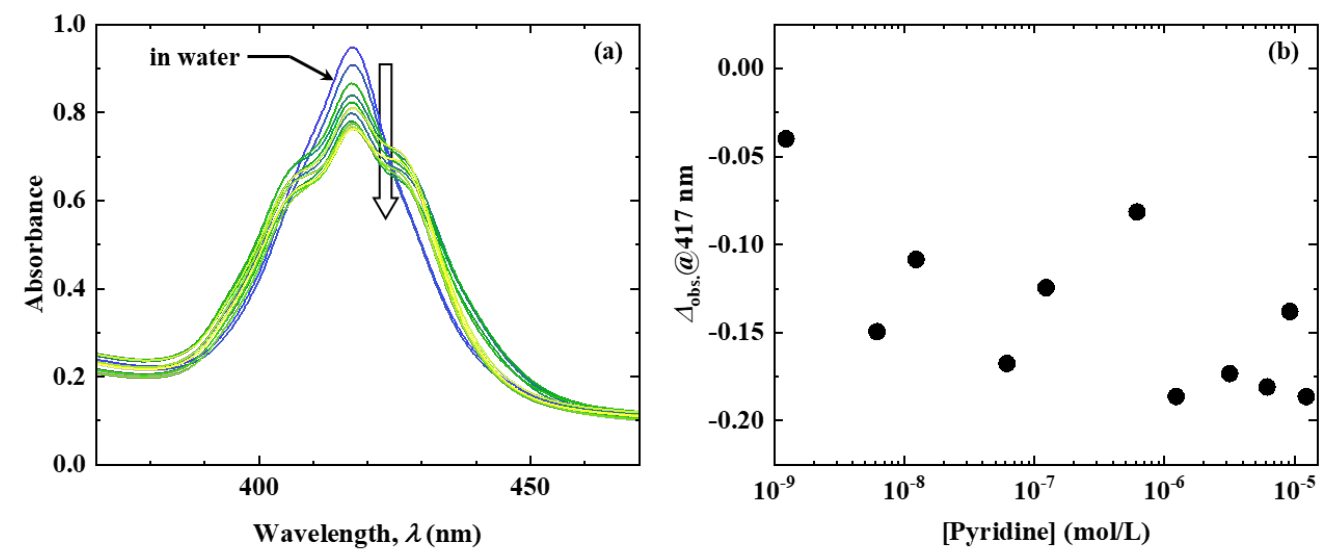

Figure 6. (a) Absorption spectrum of the transparent ZnTPPS/C10S/LDH film after immersion in various solutions of low [Pyridine] ([Pyridine] $=1.23 \mathrm{nmol} / \mathrm{L}$ to $12.3 \mu \mathrm{mol} / \mathrm{L})$. (b) Dependence of $\Delta A_{\text {abs. }}$. on [Pyridine] at $417 \mathrm{~nm}$.

the transparent ZnTPPS/C10S/LDH film can quantitatively detect pyridine in aqueous solutions at extremely low [Pyridine] (in the order of nanomoles per litre), indicating that the pyridine sensing performance of the transparent ZnTPPS/C10S/LDH film surpassed the molecular detection abilities of previously reported sensors. ${ }^{22,23}$ Furthermore, the pyridine detection ability of this transparent $\mathrm{ZnTPPS} / \mathrm{C10S} / \mathrm{LDH}$ film in an aqueous solution is comparable to that of the evanescent wave infrared chemical sensing method that achieves highly sensitive detection. Although the isosbestic point was not observed at extremely low [Pyridine], new absorption bands appeared at approximately 405 and $425 \mathrm{~nm}$. This may be attributed to the changes in both the polarity of the interlayer space of ZnTPPS/C10S/LDH and the intermolecular interactions among the ZnTPPS molecules induced by the entry of pyridine into the interlayer space of ZnTPPS/C10S/LDH, and not the formation of Py-ZnTPPS. However, further investigation of band assignment is necessary due to the fact that the mechanistic details underlying this behaviour remain unclear. In future, various experiments will be performed to clarify the pyridine detection mechanism at extremely low [Pyridine] and to improve the quantitative detection of pyridine in aqueous solutions at extremely low [Pyridine].

\section{Conclusions}

In this study, we investigated the pyridine detection performance of a transparent ZnTPPS/C10S/LDH film, which was prepared by hybridizing zinc meso-tetra (4sulfonatophenyl)porphyrin (ZnTPPS) and 1-decanesulfonate (C10S) with a transparent layered double hydroxide $(\mathrm{LDH})$ film prepared by the filtration membrane transfer method in an aqueous solution. The ZnTPPS incorporated in the transparent ZnTPPS/C10S/LDH film formed a 1:1 complex with pyridine, and the formation of Py-ZnTPPS on the transparent ZnTPPS/C10S/LDH film occurred at a pyridine concentration $(12.3 \mu \mathrm{mol} / \mathrm{L})$ that was 100 times lower than that at which the Py-ZnTPPS 
complex was formed in an aqueous solution. The equilibrium constant $\left(K_{11}\right)$ of the Py-ZnTPPS formation reaction on the transparent ZnTPPS/C10S/LDH film in an aqueous solution was 260 times that observed in an aqueous solution of ZnTPPS. Furthermore, changes were observed in the absorption spectra of the transparent ZnTPPS/C10S/LDH film at extremely low pyridine concentrations (i.e., between $1.23 \mathrm{nmol} / \mathrm{L}$ and $12.3 \mu \mathrm{mol} / \mathrm{L}$ ), which demonstrated the ability of the transparent ZnTPPS/C10S/LDH film to quantitatively detect pyridine at extremely low concentrations. These results therefore indicate that the synthesized transparent ZnTPPS/C10S/LDH film is an effective molecular sensing material for the quantitative detection of pyridine in aqueous solutions with ultra-high sensitivity, as evidenced by the change in the absorbance at $417 \mathrm{~nm}$ (Soret band). The propensity of metalloporphyrins to exhibit specific axial coordination patterns depending on the central metal species has thus been exploited to prepare a transparent film by hybridizing a metalloporphyrin with an LDH modified by amphiphilic compounds for the selective axial coordination reaction with pyridine, a toxic species. Such films have the potential to detect toxic coordinate compounds of low polarity in various media. Based on the results presented herein, we are investigating the highly sensitive and selective detection of toxic coordination molecules, such as amines, thiols, and inorganic gases using metalloporphyrins with various central metal atoms, such as $\mathrm{Fe}, \mathrm{Co}$, and precious metals incorporated in the interlayer space of $\mathrm{LDH}$. In the future, we aim to develop improved design strategies for the construction of high-sensitivity molecular detection materials using the interlayer 2D nano-space of LDHs.

\section{Materials and methods}

Materials. The $\mathrm{LDH}\left(\left[\mathrm{Mg}_{3} \mathrm{Al}(\mathrm{OH})_{8}\right]\left(\mathrm{CO}_{3}\right)_{0.5} \cdot n \mathrm{H}_{2} \mathrm{O}\right.$, anion-exchange capacity (AEC): $\left.3.25 \mathrm{mmol} / \mathrm{g}\right)$ was gifted by Kyowa Chemical Industry Co., Ltd. (Sakaide, Japan). ZnTPPS (Frontier Scientific Chemicals, Utah, USA) was used as an anionic metalloporphyrin dye without further purification. C10S (Tokyo Chemical Industry Co., Ltd. Tokyo, Japan) was used as a modifier for the LDH interlayer space without further purification. $\mathrm{NaCl}$ (FUJIFILM Wako Pure Chemicals Corporation, Osaka, Japan), $\mathrm{CH}_{3} \mathrm{COOH}$ (AcOH; Nacalai Tesque, Inc. Kyoto, Japan), $\mathrm{CH}_{3} \mathrm{COONa} \cdot 3 \mathrm{H}_{2} \mathrm{O}$ (AcONa, Kishida Chemical Co., Ltd. Osaka, Japan), $\mathrm{CH}_{3} \mathrm{CH}_{2} \mathrm{COONa} \cdot 3 \mathrm{H}_{2} \mathrm{O}$ (PrONa, Tokyo Chemical Industry Co., Ltd. Tokyo, Japan), ethanol (EtOH: FUJIFILM Wako Pure Chemicals Corporation Osaka, Japan), concentrated sulfuric acid (FUJIFILM Wako Pure Chemicals Corporation Osaka, Japan), methanol (MeOH: FUJIFILM Wako Pure Chemicals Corporation, Osaka, Japan), and $\mathrm{HClO}_{4}$ (Kanto Chemical Co., Inc. Tokyo, Japan) were used as received without further purification. Pyridine (FUJIFILM Wako Pure Chemicals Corporation, Osaka, Japan) was used as the target compound. A cover glass (dimensions: $24 \mathrm{~mm} \times 24 \mathrm{~mm}$, thickness: $0.13-0.17 \mathrm{~mm}$, Matsunami Glass Ind., Ltd. Kishiwada, Japan) was used as the glass substrate. The glass substrate was sonicated in water for $30 \mathrm{~min}$ and then treated with concentrated sulfuric acid overnight at $25{ }^{\circ} \mathrm{C}$. Subsequently, the glass substrate was 
washed with a sufficient amount of water. The water used in this research was Milli-Q water obtained from a Direct-Q UV system (Merck KGaA, Damstadt, Germany).

Preparation of transparent LDH films based on C10S and ZnTPPS. A flow chart of the procedure employed to prepare the transparent LDH film based on C10S and ZnTPPS (transparent ZnTPPS/C10S/LDH film) is shown in Figure S2 in the Supporting Information. An aqueous dispersion of the LDH nanosheet was initially prepared by dispersing the LDH powder-propionic acid mixture ( $\mathrm{PrO}^{-}-\mathrm{LDH}, 10 \mathrm{mg}$ ) in Milli-Q water $(20 \mathrm{~mL})$ under vigorous stirring. The $\mathrm{PrO}^{-}$-LDH species was synthesized via the $\mathrm{NaCl}$ /acetate-buffer decarbonation and anion-exchange methods in the presence of PrONa, as described in the literature. ${ }^{21}$ The transparent LDH film bearing carbonate anions (transparent $\mathrm{CO}_{3}{ }^{2-}$-LDH film) was prepared by applying the filtration-film transfer method to an aqueous dispersion of LDH nanosheets (total volume: $2 \mathrm{~mL}$, LDH particle concentration: $0.15 \mathrm{mg} / \mathrm{L}$ ) according to our previous report. ${ }^{19}$ The transparent LDH film bearing perchlorate anions (transparent $\mathrm{ClO}_{4}{ }^{-}$-LDH film) was prepared by immersing six pieces of the transparent $\mathrm{CO}_{3}{ }^{2-}$ - $\mathrm{LDH}$ film (size: 24 $\times 24 \mathrm{~mm})$ in an aqueous solution of $\mathrm{HClO}_{4} / \mathrm{MeOH}\left(150 \mathrm{~mL},\left[\mathrm{HClO}_{4}\right]=75.2 \mu \mathrm{mol} / \mathrm{L}\right)$ for $12 \mathrm{~h}$ under a dried $\mathrm{N}_{2}$ gas flow. The obtained transparent $\mathrm{ClO}_{4}{ }^{-}-\mathrm{LDH}$ film was then rinsed with $\mathrm{MeOH}$ and dried under reduced pressure. Subsequently, the transparent $\mathrm{ClO}_{4}{ }^{-}-\mathrm{LDH}$ film was immersed in a formamide solution containing both ZnTPPS and C10S $(10 \mathrm{~mL},[\mathrm{ZnTPPS}]=2.35 \mathrm{mmol} / \mathrm{L},[\mathrm{C} 10 \mathrm{~S}]=2.35$ $\mathrm{mmol} / \mathrm{L})$, which was purged with dried $\mathrm{N}_{2}$ gas $(0.5 \mathrm{~L} / \mathrm{min})$ for $3 \mathrm{~min}$, and stirred vigorously for $72 \mathrm{~h}$ at $25^{\circ} \mathrm{C}$. Thereafter, the obtained transparent $\mathrm{LDH}$ film based on $\mathrm{C} 10 \mathrm{~S}$ and ZnTPPS (i.e., the transparent ZnTPPS/C10S/LDH film) was rinsed with EtOH and dried under reduced pressure.

Pyridine detection ability of the transparent ZnTPPS/C10S/LDH film in aqueous media. The pyridine detection ability of the transparent ZnTPPS/C10S/LDH film was evaluated as follows. Initially, the dried transparent ZnTPPS/C10S/LDH film was immersed in aqueous pyridine solutions of various concentrations ([Pyridine] $=0-0.123 \mathrm{~mol} / \mathrm{L}$ ) for $2 \mathrm{~min}$ (step 1). After removing the transparent ZnTPPS/C10S/LDH film from the aqueous pyridine solution, the film was dried by blowing with dried $\mathrm{N}_{2}$ gas (step 2). The absorption spectrum of the transparent ZnTPPS/C10S/LDH film was then obtained using a UV-Vis spectrophotometer (V-670, JASCO, Tokyo, Japan) (step 3). The transparent ZnTPPS/C10S/LDH film was rinsed twice with EtOH and then dried by blowing with dried $\mathrm{N}_{2}$ gas (step 4). Steps 1-4 were repeated at different [Pyridine]. As a reference, the absorption spectra of ZnTPPS in aqueous pyridine solutions with various [Pyridine] were also analysed.

Characterization. The XRD analysis of each transparent film was performed on a MiniFlex II powder $\mathrm{X}$-ray diffractometer (Rigaku, Tokyo, Japan) equipped with an Ni-filtered $\mathrm{Cu}-\mathrm{K} \alpha$ radiation source (30 $\mathrm{kV}, 15 \mathrm{~mA}$; scan rate: $1 \% \mathrm{~min}$; sampling step: $0.02^{\circ}$ ). Infrared spectroscopy studies were performed on 
each transparent film using the attenuated total reflection (ATR) method on an FT/IR 6100 spectrometer (JASCO, Tokyo, Japan). The amount of ZnTPPS incorporated in the transparent ZnTPPS/C10S/LDH film was determined from the absorption spectrum of its dimethyl sulfoxide (DMSO) solution, which was prepared by dissolving the residual ZnTPPS collected by evaporation in DMSO. The PL spectra of the transparent ZnTPPS/C10S/LDH film were measured using a spectrofluorometer (FP-6600, JASCO, Tokyo, Japan) at room temperature under ambient conditions. The AFM images were acquired using a scanning probe microscope (SPM-9700, Shimadzu, Kyoto, Japan).

\section{Acknowledgements}

This work was partially supported by two programs (2010 and 2013) funded by the Shimane Industrial Promotion Foundation and the Cosmetology Research Foundation.

\section{Author contributions}

R. S. and T. F. designed the study and wrote the manuscript. Y. A. performed most of the sample preparation and measurements. All authors discussed the results and commented on the manuscript.

Competing interests: The authors declare no competing financial interests.

\section{References}

[1] https://www.mofa.go.jp/mofaj/gaiko/oda/sdgs/pdf/000101401.pdf

[2] Arshak, K., Moore, E., Lyons, G. M., Harris, J. \& Clifford, S. A review of gas sensors employed in electronic nose applications. Sens. Rev. 24, 181-198 (2004).

[3] Lee, H., Hong, K-I. \& Jang, W-D. Design and applications of molecular probes containing porphyrin derivatives. Coord. Chem. Rev. 354, 46-73 (2018).

[4] Sasai, R. et al. Luminescence Properties of Rhodamine 6G Intercalated in Surfactant/Clay Hybrid Thin Solid Films. Langmuir 20, 4715-4719 (2004).

[5] Sasai R., Hotta, Y. \& Itoh, H. Preparation of organoclay having titania nano-crystals in interlayer hydrophobic field and its characterization. J. Ceram. Soc. Jpn. 116, 205-211 (2008).

[6] Sasai, R., Itoh, T., Ohmori, W., Itoh, H. \& Kusunoki, M. Preparation and Characterization of Rhodamine 6G/Alkyltrimethylammonium/Laponite Hybrid Solid Materials with Higher Emission Quantum Yield. J. Phys. Chem. C 113, 415-421 (2009).

[7] Sasai, R. Possibility of Layered Inorganic/Dye Hybrid Solids as Molecular Sensor. Mater. Sci. Tech. Jpn. 46, 112-116 (2009).

[8] Sasai, R. Molecular Sensing Ability of Layered Inorganic/Luminous Organic Nano Hybrid Solid Materials. IOP Conf. Series: Materials Science and Engineering 18, 082003 (2011). 
[9] Sasai, R., Iyi, N. \& Kusumoto, H. Luminous Change of Rhodamine 3B Incorporated into Titanate Nanosheet/Decyltrimethylammonium Hybrids under Humid Atmosphere. Bull. Chem. Soc. Jpn. 84, 562-568 (2011).

[10] Fujimura, T. et al. High Density Intercalation of Porphyrin into Transparent Clay Membrane without Aggregation. Langmuir 29, 5060-5065 (2013).

[11] Sasai, R., Miyanaga, H. \& Morita, M. Preparation and characterization of highly photoluminescent hybrid solids from anionic fluorescein dye, surfactant, and layered double hydroxide. Clay Sci. 17, 35-40 (2013).

[12] Yui, T. et al. Intercalation of a Surfactant with a Long Polyfluoroalkyl Chain into a Clay Mineral: Unique Orientation of Polyfluoroalkyl Groups in Clay Layers. Langmuir 29, 10705-10712 (2013).

[13] Sasai, R., Watanabe, R. \& Yamada, T. Preparation and characterization of titania- and organopillared clay hybrid photocatalysts capable of oxidizing aqueous bisphenol A under visible light. Appl. Clay Sci. 93-94, 72-77 (2014).

[14] Date, Y. et al. Preparation and characterization of layered double hydroxide/anionic fluorescein dye hybrid thin solid films with luminous properties. Clay Sci. 19, 85-90 (2015).

[15] Sasai, R. \& Morita, M. Luminous relative humidity sensing by anionic fluorescein dyes incorporated into layered double hydroxide/1-butanesulfonate hybrid materials. Sens. Actuators B Chem. 238, 702-705 (2017).

[16] Date, Y. et al. Influence of Gas Adsorption on the Luminous Properties of Layered Double Hydroxide/Anionic Fluorescence Dye Hybrid Thin Solid Films. Bull. Chem. Soc. Jpn. 90, 148153 (2017).

[17] Aoyama, Y., Fujimura, T. \& Sasai, R. Preparation of $\alpha$-Zirconium Phosphate Nanosheet Stacked Solid Thin Films with High Transparency and Intercalating with Cationic Free-Base Porphyrins. Chem. Lett. 48, 40-42 (2019).

[18] Fujimura, T., Aoyama, Y. \& Sasai, R. Unique protonation behavior of cationic free-base porphyrins in the interlayer space of transparent solid Films comprising layered $\alpha$-zirconium phosphate. Tetrahedron Lett. 160, 150912 (2019).

[19] Fujimura, T., Akagashi, Y., Aoyama, Y. \& Sasai, R. Preparation of Transparent Film of Layered Double Hydroxide with Anionic Pyrene Derivatives and Its Luminous Toluene Detection Ability. Int. J. Photoenergy 2020, 8870930 (2020).

[20] Sasai, R., Yamamoto, S. \& Naito, A. Photoluminescence Gas Sensing by Fluorescein-Dye Anions/1-Butanesulfonate/Layered Double Hydroxide Hybrid Materials under Humid Environmental Conditions. Nanomaterials 11, 914 (2021).

[21] Huang, G. G., Lee, C-J., Yang, J., Chang, C-H., Sathiyendiran, M., Lu, Z-Z. \& Lu, K-L. RheniumBased Molecular Trap as an Evanescent Wave Infrared Chemical Sensing Medium for the 
Selective Determination of Amines in Air. ACS Appl. Mater. Interface 8, 35634-35640 (2016).

[22] Li, Z., Ma, J., Zong, Y. \& Men, Y. ZnS Nanoparticles for high-sensitive fluorescent detection of pyridine compounds. J. Alloys Compd. 559, 39-44 (2013).

[23] Lv, B., Cheng, C., Mo, Y., Yuan, H. \& Xiao, D. A simple strategy for pyridine visual sensing by the in-situ formation of tetranuclear copper iodine pyridine microcrystalline film on copper foil. Thin Solid Films 516, 7812-7815 (2008).

[24] Iyi, N., Ebina, Y. \& Sasaki, T. Water-Swellable MgAl-LDH (Layered Double Hydroxide) Hybrids: Synthesis, Characterization, and Film Preparation. Langmuir 24, 5591-5598 (2008).

\section{Figure legends}

Figure 1. Photographic images of the (a) $\mathrm{CO}_{3}{ }^{2-}-\mathrm{LDH}$, (b) $\mathrm{ClO}_{4}{ }^{-}-\mathrm{LDH}$, and (c) ZnTPPS/C10S/LDH transparent films. (d) AFM image of the transparent ZnTPPS/C10S/LDH film. (e) XRD patterns of the $\mathrm{CO}_{3}{ }^{2-}$-LDH (black solid line), $\mathrm{ClO}_{4}{ }^{-}-\mathrm{LDH}$ (red solid line), and $\mathrm{ZnTPPS} / \mathrm{C} 10 \mathrm{~S} / \mathrm{LDH}$ transparent films (blue solid line). (h) FT-IR spectra of the $\mathrm{CO}_{3}{ }^{2-}-\mathrm{LDH}$ (black solid line), $\mathrm{ClO}_{4}{ }^{-}-\mathrm{LDH}$ (red solid line), and transparent ZnTPPS/C10S/LDH films (blue solid line). Arrows indicate the peaks originating from the hydroxyl (black), carbonate (black (open)), methylene (green), perchlorate (red (open)) and sulfonate (blue (open)) groups. (g) Schematic structural model of the ZnTPPS/C10S/LDH materials.

Figure 2. (a) Absorption spectrum of the transparent ZnTPPS/C10S/LDH film. (b) PL spectra of the transparent ZnTPPS/C10S/LDH film at excitation wavelengths of $410(\square), 420(\bigcirc), 430$ (solid line), 440 (broken line), and 450 (dotted line) $\mathrm{nm}$.

Figure 3. Absorption spectra of the ZnTPPS/C10S/LDH transparent film after immersion in solutions of various [Pyridine] $([$ Pyridine $]=12.3 \mu \mathrm{mol} / \mathrm{L}$ to $0.123 \mathrm{~mol} / \mathrm{L})$.

Figure 4. Dependence of $\Delta A_{\text {obs. }}$ on [Pyridine] for the transparent ZnTPPS/C10S/LDH film $(\bigcirc, 429 \mathrm{~nm})$ and ZnTPPS ( $\square, 425 \mathrm{~nm}$ ) in an aqueous solution. The broken lines represent the fitting curves estimated using Equation (1).

Figure 5. Schematic structural model of the ZnTPPS/C10S/LDH materials in the (a) absence and (b) presence of pyridine in an aqueous solution.

Figure 6. (a) Absorption spectrum of the transparent ZnTPPS/C10S/LDH film after immersion in various solutions of low [Pyridine] $\left([\right.$ Pyridine $]=1.23 \mathrm{nmol} / \mathrm{L}$ to $12.3 \mu \mathrm{mol} / \mathrm{L}$ ). (b) Dependence of $\Delta A_{\text {abs. }}$ on [Pyridine] at $417 \mathrm{~nm}$. 


\section{Supplementary Files}

This is a list of supplementary files associated with this preprint. Click to download.

- SupportingInformation.docx 